\title{
Cómo incorporar las estrategias de control de la comprensión a la enseñanza con textos base de lectura *
}

\author{
Maribeth Cassidy Schmitt \\ James F. Baumann
}
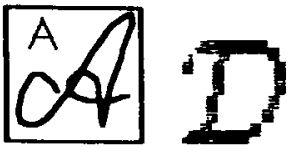

Como la vieja sabiduria de Confucio nos recuerda desde hace miles de años, el saber más importante no es el conocimiento en sí, sino lo que boy denominamos el meta-conocimiento: saber lo que se sabe y saber lo que no se sabe. La decodificación no reflexionada del alumno, no interpretada o no situada conscientemente en sus esquemas previos, produce una comprensión muy molecular y un recuerdo débil. Pero cómo conseguir desarrollar esta meta-comprensión, ese conocimiento más amplio y sólido en el niño. ¿Qué métodos y recursos utilizar para lograrlo? Cassidy y Baumann los exponen aqui con gran claridad.

La conciencia y el control que tiene el individuo sobre los procesos cognitivos implicados en la atención, comprensión y recuerdo de la información, se han relacionado con la metacognición (por ejemplo: Flavell, 1976; Baker y Brown, 1984). En lo relativo a la lectura, la metacognición se ha denominado «metacomprensión» e implica el proceso de control de la comprensión y la utilización de estrategias independientes que faciliten el aprendizaje y el recuerdo (Baker y Brown, 1984).

No se dispone de evidencia empírica alguna que sugiera que, en las clases de hoy en día, se fomenta la conciencia metacognitiva o que existan prácticas educativas tendentes al desarrollo de la metacomprensión. Sin embar-

"How to incorporate comprehension monitoring strategies into basal reader instruction". The Reading Teacher, 1986, 40 (1), pp. 28-31. Reproducida con autorización de M. Cassidy Schmitt y James F. Baumann y de la International Reading Association (C). De la traducción al castellano CLEE (Traducción de Patricia Fernández. La I.R.A. no se responsabiliza de la adecuación de la traducción).

* "Basal reader», en inglés, corresponde al libro de lectura de curso que se utiliza como apoyo del libro de lenguaje. 


\section{6}

go, los resultados de diversos estudios indican que es perfectamente posible entrenar a los estudiantes para que desarrollen actividades metacognitivas. Por ejemplo, los estudiantes demostraron que mejoraba su comprensión después de que se les enseñaba a generar sus propias preguntas basadas en el esquema de la historia (Singer y Donlan, 1982). Palincsar y Brown (1983), establecieron que mejoraba la comprensión cuando los estudiantes y el profesor se turnaban para "entregar» aquella información relativa a los pasajes por medio de un resumen, preguntas, aclaración y predicción.

Sin embargo, hasta el momento, se ha dado poca importancia al hecho de cómo mejorar la enseñanza utilizando libros de lectura de curso tradicionales para promover y desarrollar las actividades metacognitivas. El propósito de este artículo, es describir cómo el profesor puede integrar el uso de estrategias de control de la comprensión en la etapa de lectura dirigida cuando emplea libros de lectura de curso.

\section{EL CONTROL DE LA COMPRENSION Y LOS LIBROS DE LECTURA DE CURSO: UN EJEMPLO}

La metacomprensión tiene lugar cuando los estudiantes son conscientes de la importancia de la actividad lectora. Específicamente, cuando los estudiantes se dan cuenta de los beneficios que acarrea el uso de la estrategia, se muestran más inclinados a utilizarlas independientemente de las sugerencias del profesor. Esto último coincide con las ideas de Paris, Lipson y Wixson (1983), de que la conducta lectora estratégica depende de la autoselección intencionada de una estrategia como medio para conseguir un fin. La enseñanza de cualquier estrategia lectora debería incorporar, esencialmente, lo que estos autores denominan "conocimiento condicional", es decir, saber cuándo se utiliza una estrategia y el por qué es relevante o importante hacerlo.

La actividad de lectura dirigida que se emplea en la enseñanza de selecciones básicas, puede modificarse con el objeto de instruir a los estudiantes para que se familiaricen con las diversas estrategias de control de la comprensión. Concretamente, es posible entrenar a los estudiantes para que activen sus conocimientos básicos, formulen preguntas, hagan predicciones, establezcan propósitos para la lectura y resuman lo que han leído. Al principio, el profesor debe guiar a sus alumnos en estas actividades pero, gradualmente, los estudiantes deberían asumir tales responsabilidades (Baumann, 1983; Pearson, 1985) y ponerlas en práctica de manera independiente cuando leen una historia. La siguiente lección ilustra cómo puede implementarse el ejemplo anterior. Esta lección incluye la lectura dirigida de la historia «La reina que no sabía hornear pan de jengibre», extraída de un libro de lectura de tercero de E.G.B. (Clymer et al., 1985). La sinopsis de la historia que se cita a continuación es una descripción de las diversas actividades que podría elegir un profesor para aumentar el control de la comprensión a través de las fases de pre-lectura, lectura y post-lectura en la actividad de lectura dirigida.

Sinopsis de la bistoria: «La reina que no sabía hornear pan de jengibre», es un cuento fantástico sobre el Rey Pilaf que decidió que había llegado la 
hora de buscar una esposa. Tomó esa decisión porque se sentía solo, las cosas no le iban muy bien y porque estaba cansado de comer un mal pan de jengibre para el desayuno, todas las mañanas. Había decidido que su reina debía ser bella, inteligente y capaz de hornear pan de jengibre. En otro pueblo, la Princesa Caliope se encontraba buscando un marido que fuera rey, atractivo y capaz de tocar el trombón; algo que el Rey Pilaf no podía hacer. Después de un tiempo, ambos decidieron comprometerse, casarse, y nunca mencionar el pan de jengibre ni el trombón. Así vivieron felices hasta que un mal día el rey quiso comer pan de jengibre y la reina escuchar el trombón. Entonces riñeron y se fueron a vivir a rincones opuestos del castillo. Tiempo después, volvieron a juntarse, luego de considerar sus prioridades reales. Para entonces, el rey había aprendido a hornear pan de jengibre y la reina a tocar el trombón.

\section{ACTIVIDADES DE PRE-LECTURA}

\section{Activar el conocimiento básico}

Para ayudarles a comprender la historia, diga a los estudiantes que la comprensión es más fácil si utilizan lo que ya conocen sobre el tema. Con el fin de ayudarles a pensar en lo que ya saben sobre reyes, reinas, reinos y pan de jengibre, dígales que pueden utilizar los dibujos y los títulos. Después de esto podría organizarse una discusión rápida sobre lo que el grupo ya conoce acerca del tema.

\section{Hacer predicciones sobre el contenido}

Explique a sus alumnos que puede resultar divertido hacer predicciones. De este modo, promoverá la comprensión cada vez que lean para evaluar sus predicciones. Anímelos a predecir diversos elementos de la historia, tales como: acciones de los personajes, problemas, posibles soluciones y desenlace. El uso de los dibujos, títulos e idea principal, pueden servir nuevamente a la hora de elaborar posibles predicciones. En el caso de la historia citada, por ejemplo, las predicciones podrían incluir ideas tales como: «El rey se volvió loco porque la reina no sabía hornear pan de jengibre y la echó del reino" o "La reina no sabía cómo preparar pan de jengibre, así que todos los habitantes del reino le ayudaron a aprender». Las predicciones hechas en grupo pueden grabarse, o realizarse por escrito si se hacen de forma individual.

\section{Establecer propósitos para la lectura}

Explique a sus alumnos que deberían leer la historia teniendo siempre algún objetivo en mente. Esto les ayudaría a focalizar su atención y a mejorar la comprensión activa. Las predicciones realizadas por ellos aportan una valiosa fuente a la hora de plantearse objetivos. Por ejemplo, es posible que los alumnos lean "para averiguar si el rey desterró a la reina».

\section{Generar preguntas}

Diga a sus alumnos que, antes de comenzar a leer, deberían generar preguntas a las que desearian responder. Esto les mantendrá absorbidos en la 
historia y mejorará su comprensión. Tales preguntas deberían guardar relación con los elementos generales de la narración. En el ejemplo citado anteriormente, las preguntas podrían ser: «¿Esta historia va a contar más sobre la reina mencionada en el título o acerca del rey que aparece en los dibujos? ¿Qué tipo de persona es el rey o la reina? ¿Puede constituir un problema tan grande el hecho de que la reina no sepa hornear pan de jengibre? ¿Y si así fuera, cómo podría solucionarse?»

\section{ACTIVIDADES DE LECTURA DIRIGIDA}

\section{Resumir en diversos puntos}

Explique a los alumnos que es aconsejable interrumpir la lectura, cada cierto tiempo, con el fin de revisar las ideas principales. Esta modalidad resulta excelente para comprobar si se ha ido comprendiendo a medida que se lee. Los resúmenes deberían hacerse en aquellos puntos donde se desarrollan los diversos elementos estructurales. En el caso de la historia citada como ejemplo, podría hacerse un resumen después de que se ha presentado al rey y descrito el contexto; y otro, después de que se han explicado los problemas del rey y lo que él está haciendo para solucionarlos.

\section{Evaluar y hacer nuevas predicciones}

Diga a los estudiantes que, a medida que avancen en la lectura de la historia, deberán ser capaces de ir evaluando sus predicciones basándose en la nueva información que vayan obteniendo, así como de aclarar cualquier problema de comprensión. También, deberían ser capaces de adecuar las predicciones realizadas o de hacer otras nuevas a medida que leen y descubren nuevos datos. Esta actividad permite mantener animadas discusiones en base a la información que se va obteniendo. Por ejemplo, en esta historia, los estudiantes podrían darse cuenta que su predicción sobre el destierro de la reina por parte del rey era incorrecta y que podrían hacer una nueva diciendo si el rey se casará o no con la princesa, aunque no sepa hornear pan de jengibre.

\section{Relacionar la nueva información con el conocimiento previo}

Explique a sus alumnos que sólo con que piensen en lo que ya sabían sobre reyes, reinas y pan de jengibre, al comienzo de la historia, mejorará su comprensión. Haciendo lo mismo con cada una de las nuevas ideas que aparezcan les resultará mucho más fácil comprender el texto. Por ejemplo, cuando la princesa dice que su marido debería saber tocar el trombón, los alumnos deberían activar sus esquemas sobre «instrumentos musicales».

\section{Generar preguntas}

Diga a los alumnos que no siempre contarán con el profesor para que responda y aclare las dudas que se les van planteando a lo largo de la lectura. Anímeles a formùlar preguntas apropiadas durante el transcurso de la historia, tales como: «¿Cómo conoció el rey a la princesa Calíope? ¿Cómo era ella? ¿Qué buscaba la princesa?» Aunque estas preguntas son bastante 
similares a las que aparecen en el manual del profesor, la responsabilidad de formularlas recae, en este caso, directamente sobre los estudiantes, que aprenden a procesar las historias de una manera independiente.

\section{ACTIVIDADES DE POST-LECTURA}

\section{Resumir la selección completa}

Para mejorar la comprensión y recordar lo que se ha leído, enseñe a sus alumnos a resumir la selección completa relatando solamente los puntos principales. Esta actividad puede realizarse eleborando resúmenes orales en grupo o de forma individual mediante resúmenes escritos.

\section{Evaluar las predicciones}

Los alumnos deberían emplear toda la información que aparece en la historia, para evaluar sus predicciones, discutir acerca de cómo mejorarlas y comentar aquellos datos que les llevaron a cambiarlas. Un punto clave, en el caso del ejemplo citado, es que el título les sugiere de inmediato algunas cosas relacionadas con la reina cuando, de hecho, la mayor parte de la historia versa sobre el rey y sus necesidades.

\section{Volver a establecer propósitos para la lectura}

Señale a los alumnos que la mejor manera de comprobar si han comprendido es juzgar al final si están satisfechos o no con el propósito que establecieron al principio de la lectura. En este caso, ¿averiguaron por fin lo que sucedió con la reina que no sabia hornear pan de jengibre?

\section{Generar preguntas para la selección total}

En este punto, el profesor ya puede compartir con los alumnos la responsabilidad de generar preguntas. Ellos deberían aprender a formular preguntas tendentes a recoger información sobre los personajes, el problema, el objetivo y la solución. Pueden formular las preguntas a algún compañero del grupo o al profesor. El éxito o fracaso que tengan en esta tarea puede aportarles una valiosa información sobre la comprensión global.

\section{CONCLUSION}

Estas actividades promueven la comprensión de los alumnos, con el sólo requisito de que sean sujetos activos. La organización es la misma que se emplea en los manuales de lectura, es decir, actividades de prelectura, una lectura dirigida de la historia y una discusión al final de la lectura. El aspecto innovador de este programa radica en que los estudiantes asumen la mayor responsabilidad en la comprensión y control de la comprensión, a diferencia de otros en que la comprensión de los alumnos es responsabilidad absoluta del profesor. 


\section{Referencias}

BAKER, LINDA, y ANN L. BROWN. «Metacognitive Skills and Reading.* In Handbook of Reading Research, editado por P. David Pearson Nueva York, N.Y.: Longman, 1984.

BaumanN, JAMES F. *A Generic Comprehension Instructional Strategy.* Reading World, vol. 22, mayo 1983, pp. 284-94.

Clymer, Theodore; Roselmina Indrisano; Dale D. Johnson; P. David Pearson, y RiCHARD Venezky. The Ginn Reading Program, Level 10, Lexington, Mass.: Ginn and Company, 1985.

FlavelL, J. H. *Metacognitive Aspects of Problem Solving.* In The Nature of Intelligence, editado por L. B. Resnick. Hillsdale, N.J.: Lawrence Erlbaum Associates, 1976.

PALINCSAR, ANNEMARIE S., y ANN BROWN. «Reciprocal Teaching of Comprehension-Monitoring Activities.» Technical Report No. 269. Champaign, III.: Center for the Study of Reading, University of Illinois, 1983.

PARIS, SCOTT G.; MARJORIE Y. LIPSON, y KAREN K. WIXSON. *Becoming a Strategic Reader.s Contemporary Educational Psychology, vol. 8 (julio 1983), pp. 293-316.

PeARSON, P. David. «Changing the Face of Reading Comprehension Instruction.» The Reading Teacher, vol. 38 (abril 1985), pp. 724-38.

Singer, Harry, y DAN DOLAN. «Active Comprehension: Problem Solving Schema with Question Generation for Comprehension of Complex Short Stories.» Reading Research Quarterly, vol. 17, n. 2, 1982, pp. 166-84.

\section{Datos sobre los autores}

Schmitt es estudiante de doctorado e instructora licenciada en lectura y diseño educativo de la Universidad de Purdue, en West Lafayette, Indiana. Baumann es, a su vez, profesor e investigador en comprensión lectora de la Clínica de Lectura en la misma Universidad. 